Check for updates

Cite this: RSC Adv., 2017, 7, 55846

\title{
Effect of meteorological factors on photovoltaic power forecast based on the neural network
}

\begin{abstract}
Wenbo Xiao, (D) *a Jin Dai, ${ }^{a}$ Huaming Wu, ${ }^{a}$ Gina Nazario ${ }^{b}$ and Feng Cheng*b
In this paper, the effects of meteorological factors (including air temperature, wind speed, and relative humidity) on photovoltaic (PV) power forecast using neural network models have been studied. The research is based on PV power data collected at Nanchang. China. Our results showed that prediction results of three neural network models were overall close to the experimental data. It indicated the accuracy of the neural network approach. The time-power curves showed that the prediction errors were relatively large for some time frames, especially at dusk. The SSE/MSE and the coefficients of determination analysis showed that the model including air temperature had the strongest correlation with experimental data than another 2 models including wind speed and relative humidity, which proves that air temperature is an important factor for predicting the output power of PV cells.
\end{abstract}

Received 25th September 2017 Accepted 30th November 2017

DOI: 10.1039/c7ra10591f

rsc.li/rsc-advances

A neural network model has a lot of advantages. First, it does

\section{Introduction}

The burning of fossil fuels, including coal, oil, and natural gas, causes pollution that not only can harm health, but also foster climate change. Thus, there is unprecedented interest in renewable energy, especially photovoltaic (PV) power generation. ${ }^{1,2}$ However, the power output from PV power plants is influenced by the weather conditions, so it has the shortcomings of intermittence and volatility. Photovoltaic power forecasting technologies have been widely studied in order to reduce the negative impacts of photovoltaic power on the existing grid. ${ }^{3,4}$ In all proposed methods, the neural network method has drawn the attention of researchers. Neural network models are employed in the field of artificial intelligence with the aim of reproducing activities typical of the human brain. The basic structure of a neural network model has typically many input units, one or more layers of hidden units and only one output unit. Inputs are fed in from the left, hidden units are activated in the middle, then output feeds exit through the right. All the units are connected, including the layers on both sides. The weight of the connection between any two units is gradually adjusted as the network learns. For transforming input units into output units, a transfer function should be selected. The transfer function is a monotonically increasing, continuous, differentiable function, applied to the weighted input of a neuron to produce the final output.

ajiangxi Engineering Laboratory for Optoelectronics Testing Technology, Nanchang Hangkong University, Nanchang 330063, China. E-mail: xiaowenbo1570@163.com

${ }^{b}$ Department of Pharmaceutical Science, College of Pharmacy, University of South Florida, Tampa, FL, 33612, USA. E-mail: fcheng1@health.usf.edu not need many parameters or complicated calculations unlike analytical models. ${ }^{5,6}$ Second, a neural network model is able to handle missing data and solve problems with a high degree of complexity. ${ }^{7}$ Third, it was proven that neural network models perform better than polynomial regression models on photovoltaic (PV) power forecast. ${ }^{8}$ Fourth, the neural network model is easily built using the Neural Network Toolbox of Matlab that provides functions for designing, implementing, and displaying neural networks. ${ }^{9}$

The prediction accuracies of a neural network model are highly sensitive to the selection of input variables. The previous research results showed that the output of the photovoltaic power plant had a strong relationship with the irradiance intensity and the cell temperature. ${ }^{10,11}$ The relationship has been proved in theory (ref). Therefore, the entrance layer has only two nodes (the irradiance intensity and the cell temperature) in some neural network models. ${ }^{12,13}$ In fact, there are many factors, such as air temperature, ${ }^{14}$ wind speed and relative humidity, that can affect the electrical power generation of the PV systems. ${ }^{15}$ The selection of input factors is critical to identify the optimal function and increase the prediction accuracy in neural network models. ${ }^{16}$

In this paper, the effects of meteorological factors including air temperature, wind speed, and relative humidity on the PV power prediction accuracy using neural network were investigated. The PV power generation data were collected at Nanchang, China. It was shown that the predicted PV power output values of three neural network models were overall close to the measurements. The SSE/MSE and the coefficients of determination analysis showed that air temperature may be an important factor for predicting the output power of PV cells. 


\section{Material and methods}

\subsection{Experimental setup}

As shown in Fig. 1(a), the experimental setup included a PV module, an air temperature meter, a relative humidity meter, a cell temperature meter, a wind speed detector, an optical radiation detector, a Keithley 2400 digital source, and a computer. The PV module was $135 \mathrm{~mm} \times 125 \mathrm{~mm}$ polycrystalline solar cell manufactured by Guangzhou Z.T Solar Technology Co. Ltd. The module maximum output power (MOP) is $2 \mathrm{~W}$ under standard test condition. The air temperature and relative humidity were measured by a model AR807 thermometer, which was produced by Smart Sensor Co., Ltd. The cell temperature was detected by a non-contact infrared thermometer of model AR320 manufactured by Vantron Electronics Co., Ltd. The wind speed was monitored by an anemometer of model AR816, which was manufactured by Smart Sensor Co., Ltd. The irradiation intensity was measured by a meter of model HT-855, which was produced by Hong Cheng Jiuye Electronic Technology Co., Ltd. The MOP of the PV module was obtained by Keithley 2400 digital source. In the measurement process, the MOP of the PV module, optical irradiance intensity, cell temperature, wind speed, air temperature, and relative humidity were measured simultaneously in order to avoid any spurious effects. All the measurements mentioned above was automatically collected by the program in the computer. The measured time frame was from 9:00 to 18:00 during May $21^{\text {st }}, 2014$ and May $26^{\text {th }}, 2014$. The time interval for measurements was 10 minutes. Therefore, a set of 330 MOPs was collected. A set of 275 MOPs from May 21 ${ }^{\text {st }}, 2014$ to May $25^{\text {th }}, 2014$ was selected as training data. A set of 55 MOPs at May $26^{\text {th }}, 2014$ was used as prediction data. The above-mentioned forecast process was repeated 10 times.

\subsection{Neural network model}

Three neural network models were investigated in this paper. As shown in Fig. 1(b), these models consists of three layers: input, hidden, and output layer. The input layer has three nodes: the optical radiation intensity, cell temperature, and a meteorological factor (air temperature (model 1), wind speed (model 2), or relative humidity (model 3)). The output layer has one node (MOP of PV cell). According to Kolmogorov theorem, ${ }^{17}$ the number of hidden neurons was set to 7 . The tansig function between the input layer and the hidden layer was chosen as the transfer function. The purelin function between the hidden layer and the output layer was chosen as the transfer function. The learning algorithm of the proposed neural network was Levenberg-Marquardt algorithm.

\section{Results}

Fig. 2 showed the three neural network prediction results compared with measured MOP values on May $26^{\text {th }}, 2014$. The input of these neural network models has three nodes: the optical radiation intensity, cell temperature, and a meteorological factor including air temperature (model 1), wind speed (model 2), or relative humidity (model 3). The prediction results (red curves) are overall close to the experimental data (black curves) for these 3 models, which indicated the accuracy of the neural network approach for PV power forecast.

The time-power curves showed that the prediction errors were relatively large at some time frames, especially at dusk (after 17:00). At that time, the measured PV power value (close to zero) and the background noise of machines may have large effects of the measured values. Air temperature, the wind speed, and the relative humidity of the Nanchang area also change significantly at dusk. In addition, the number of extreme-lowsignal samples in the training set of neural network is small. These factors may affect the accuracy of prediction. Additionally, these models do not perform very well for some time frames close to noon (from 11:00 to 14:00). The reason may be that the environmental temperature is high and the air convection is large at that time.

In order to further understand three meteorological factors on the prediction accuracy, the sum of square error (SSE) and mean squared error (MSE) between prediction results and experimental data were calculated for models 1-3. As shown in Fig. 3, model 1 showed the minimum average SSE and MSE,

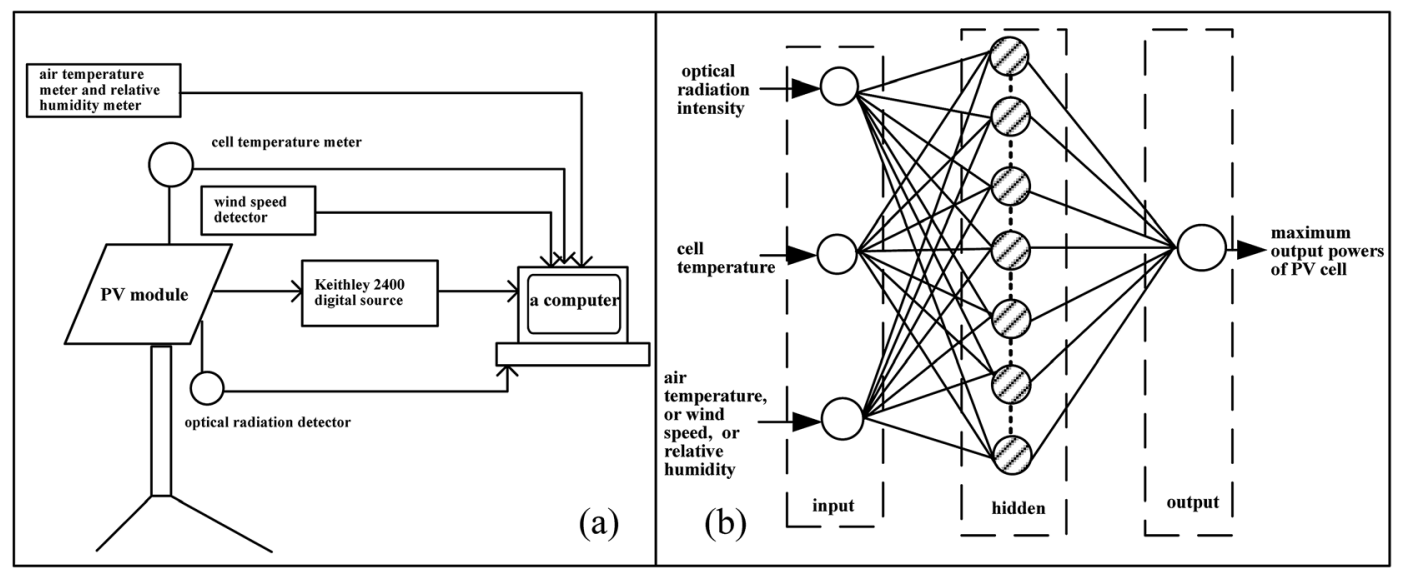

Fig. 1 Experimental setup (a) and neural network structure (b). 

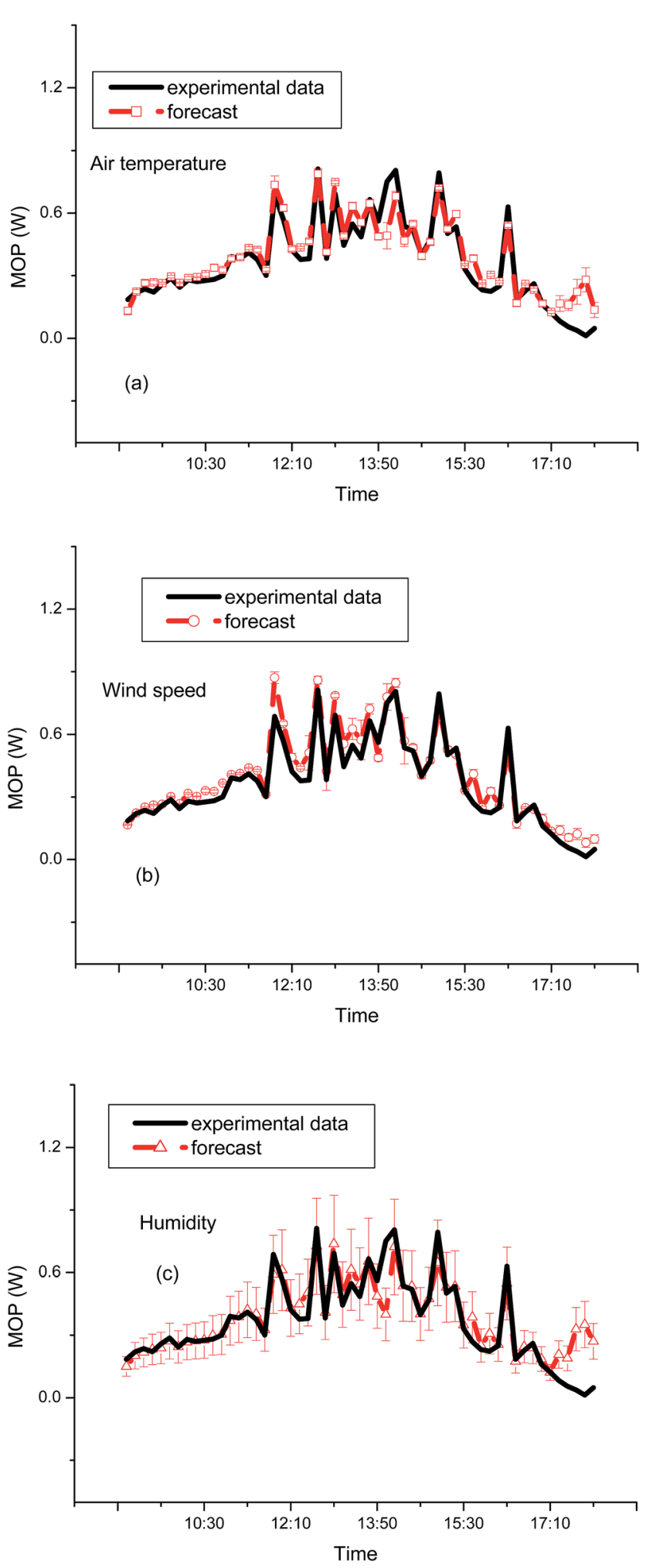

Fig. 2 Comparison of prediction results (average ten times) of three neural network models with measured values on May $26^{\text {th }}, 2014$. ((a) Model 1: the input layer consists of the optical radiation intensity, cell temperature, and air temperature; (b) model 2: the input layer consists of the optical radiation intensity, cell temperature, and wind speed; (c) model 3: the input layer consists of the optical radiation intensity, cell temperature, and relative humidity).

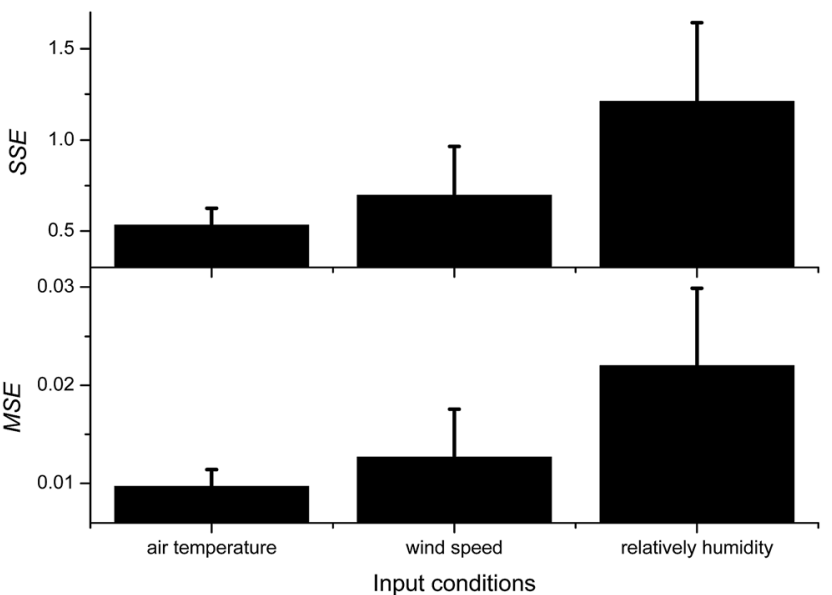

Fig. 3 The average SSE and MSE between prediction results and experimental data at May 262014 of three neural network models (air temperature (model 1 ), wind speed (model 2 ), and relative humidity (model 3)). Model 1: the input layer consists of the optical radiation intensity, cell temperature, and air temperature; model 2: the input layer consists of the optical radiation intensity, cell temperature, and wind speed; model 3: the input layer consists of the optical radiation intensity, cell temperature, and relative humidity.

while the model 3 showed the maximum average SSE and MSE. These results indicated that the model 1 had the strongest correlation with the measured POV values, followed by the model 2 , and then the model 3 . Therefore, the order of effects of meteorological factors on PV power forecasting using neural network from largest to smallest is: air temperature, wind speed, and then relative humidity. In addition, the SSE/MSE variance of model 1 also showed the same trend. We can conclude that air temperature is an important factor for PV module output power prediction using neural network.

For above calculation, data collected on May $26^{\text {th }}$ were selected as the test set. In order to check the batch (date) effects on photovoltaic power forecast, a set of 275 MOP values from any dates between May $21^{\text {st }}$, and May $26^{\text {th }}, 2014$ were randomly selected as the training set, the left 55 samples were taken as the test set. The whole procedure was also repeated 10 times for models 1-3. The coefficients of determination and SSEs/MSEs between experimental and predicted MOP values were shown in Fig. 4 and 5.

As shown in Fig. 4, the coefficients of determination for all models are above 0.84 . In general, the higher the coefficients of determination, the better the model fits the data. Therefore, there is a strong correlation between the predicted and experimental values, which prove the robust of the neural network models for PV power forecasting.

As shown in Fig. 5, for a set of 55 MOPs randomly selected from any dates between May $21^{\text {st }}$ and May $26^{\text {th }}, 2014$. The average SSE and MSE between prediction results and experimental data of models 1-3 shows similar trend as Fig. 3 . The results confirmed the conclusion that air temperature was an important meteorological factor for PV power prediction. 


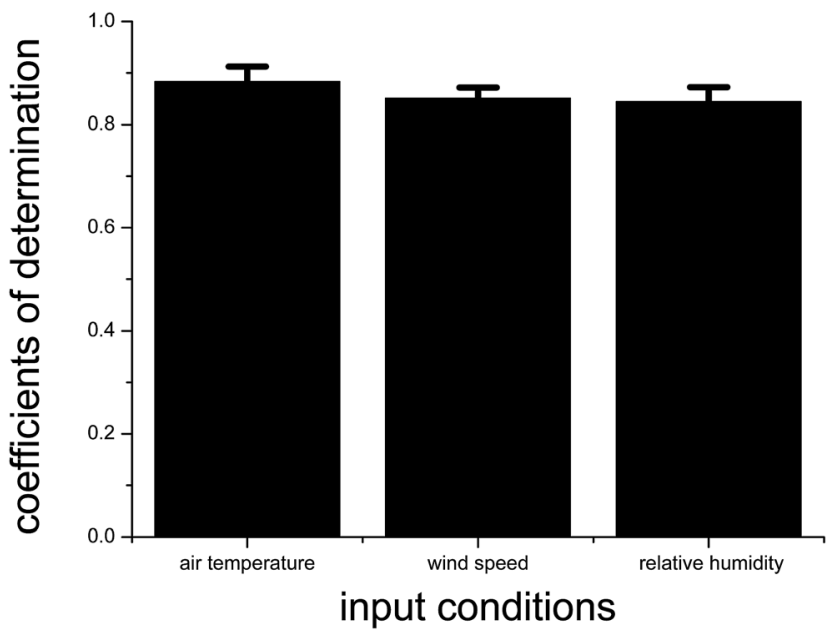

Fig. 4 The coefficients of determination between experimental and predicted data of models $1-3$ for predicting a set of 55 MOPs randomly selected from any dates between May $21^{\text {st }}$ and May $26^{\text {th }}, 2014$. Model 1: the input layer consists of the optical radiation intensity, cell temperature, and air temperature; model 2: the input layer consists of the optical radiation intensity, cell temperature, and wind speed; model 3: the input layer consists of the optical radiation intensity, cell temperature, and relative humidity.

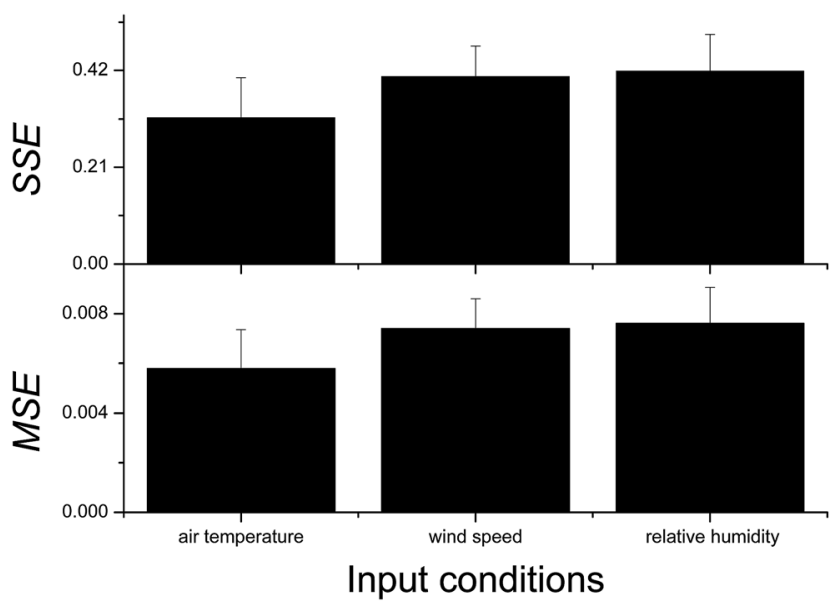

Fig. 5 The average SSE and MSE between prediction results and experimental data of models $1-3$ for predicting a set of 55 MOPs randomly selected from any dates between May $21^{\text {st }}$ and May $26^{\text {th }}$, 2014. Model 1: the input layer consists of the optical radiation intensity, cell temperature, and air temperature; model 2: the input layer consists of the optical radiation intensity, cell temperature, and wind speed; model 3: the input layer consists of the optical radiation intensity, cell temperature, and relative humidity.

\section{Conclusion}

This paper investigated the effects of the meteorological factors (including air temperature, relative humidity and wind speed) on the prediction of photovoltaic power generation using neural network models. The prediction results are overall close to the experimental data for 3 neural network models, which indicated the accuracy of the neural network approach. The time-MOP curves showed that the prediction errors were relatively large at some time frames, especially at dusk. The SSE/MSE and the coefficients of determination analysis showed the order of effects of meteorological factors on PV power forecasting using neural network from largest to smallest is: air temperature, wind speed, and then relative humidity. In the next stage, we plan to collect more samples in different conditions. We believe neural network models proposed here will be more reliable with a larger training set size.

\section{Authors' contributions}

W. X. and J. D. conceived and designed the study. W. X., J. D. and $\mathrm{H}$. W. performed the experiments. W. X. wrote the paper. G. N. and F. C. discussed, reviewed and edited the manuscript. All authors read and approved the manuscript.

\section{Conflicts of interest}

The authors declare that they have no competing financial interests.

\section{Acknowledgements}

This work was in part supported Jiangxi Province Science Major Program for Youths under Grant No. 20143ACB21011, and the Scientific Research Foundation of Jiangxi Provincial Department of Education under Grant No. GJJ150729.

\section{References}

$1 \mathrm{~J}$. Yan and B. R. Saunders, Third-generation solar cells: a review and comparison of polymer: fullerene, hybrid polymer and perovskite solar cells, RSC Adv., 2014, 4, 43286-43314.

2 P. Xu, T. Xu, J. Lu, et al. Visible-light-driven photocatalytic Sand C-codoped meso/nanoporous $\mathrm{TiO}_{2}$, Energy Environ. Sci., 2010, 3, 1128-1134.

3 B. Parida, S. Iniyan and R. Goic, A review of solar photovoltaic technologies, Renew. Sustain. Energy Rev., 2011, 15, 1625-1636.

4 J. B. Bai, Y. Cao, Y. Z. Hao, et al. Characteristic output of PV systems under partial shading or mismatch conditions, Sol. Energy, 2015, 112, 41-54.

5 T. Hiyama and K. Kitabayashi, Neural network based estimation of maximum power generation from PV module using environmental information, IEEE Trans. Energy Convers., 1997, 12(3), 241-247.

6 A. N. Celik, Artificial neural network modelling and experimental verification of the operating current of monocrystalline photovoltaic modules, Sol. Energy, 2011, 85, 2507-2517.

7 F. Grimaccia, S. Leva, M. Mussetta, et al. ANN Sizing Procedure for the Day-Ahead Output Power Forecast of a PV Plant, Appl. Sci., 2017, 7, 622. 
8 A. Mellit, S. Sağlam and S. A. Kalogirou, Artificial neural network-based model for estimating the produced power of a photovoltaic module, Renewable Energy, 2013, 60, 71-78.

9 E. Velilla, J. Valencia and F. Jaramillo, Performance evaluation of two solar photovoltaic technologies under atmospheric exposure using artificial neural network models, Sol. Energy, 2014, 107, 260-271.

10 E. Skoplaki and J. A. Palyvos, On the temperature dependence of photovoltaic module electrical performance: a review of efficiency/power correlations, Sol. Energy, 2009, 83, 614-624.

11 D. Alberto, L. Sonia and M. Giampaolo, Comparison of different physical models for PV power output prediction, Sol. Energy, 2015, 119, 83-99.

12 F. Almonacid, C. Rus, L. Hontoria, et al. Characterisation of Si-crystalline PV modules by artificial neural networks, Renewable Energy, 2009, 34, 941-949.
13 F. Almonacid, C. Rus, P. Pérez-Higueras, et al. Calculation of the energy provided by a PV generator comparative study: conventional methods vs. artificial neural networks, Energy, 2011, 36, 375-384.

14 D. Anderson, T. Sample and E. Dunlop, Obtaining module energy rating from standard laboratory measurements, in 17th European photovoltaic solar energy conference, Munich, 2001, pp. 832-35.

15 T. C. Yu and H. T. Chang, The forecast of the electrical energy generated by photovoltaic systems using neural network method, 2011 International Conference on Electric Information and Control Engineering, Wuhan, 2011, pp. 1517.

16 Y. K. Wu, C. R. Chen and H. A. Rahman, A Novel Hybrid Model for Short-Term Forecasting in PV Power Generation, Int. J. Photoenergy, 2014, 2014, 569249.

17 V. Kurkova, Kolmogorov's theorem and multilayer neural networks, Neural Network., 1992, 5, 501-506. 